\title{
Outflows from Quasars and Ultraluminous X-ray Sources
}

\author{
A.R. King ${ }^{\mathrm{a}}$ \\ ${ }^{a}$ Theoretical Astrophysics Group, University of Leicester, Leicester LE1 7RH U.K.
}

Mass outflows from Eddington-limited accreting compact objects appear to be a very widespread phenomenon. They may provide the soft excess observed in quasars and ULXs, and imply that such objects have a major effect on their surroundings. In particular they allow a simple parameter-free argument for the $M_{\mathrm{BH}}-\sigma$ relation for galaxies, and offer a straightforward interpretation of the emission nebulae seen around ULXs.

\section{INTRODUCTION}

Recent XMM-Newton observations of bright quasars (Pounds et al., 2003a, b; Reeves et al., 2003) give strong evidence for intense outflows from the nucleus, with mass rates $\dot{M}_{\text {out }} \sim$ $1 \mathrm{M}_{\odot} \mathrm{yr}^{-1}$ and velocity $v \sim 0.1 c$, in the form of blueshifted X-ray absorption lines. These outflows closely resemble those recently inferred in a set of ultraluminous X-ray sources (ULXs) with extremely soft spectral components (Mukai et al, 2003; Fabbiano et al., 2003). Simple theory shows that the outflows are probably optically thick to electron scattering, with a photosphere of $\sim 100$ Schwarzschild radii, and driven by continuum radiation pressure. In all cases the outflow velocity is close to the escape velocity from the scattering photosphere. As a result the outflow momentum flux is comparable to that in the Eddingtonlimited radiation field, i.e.

$\dot{M}_{\text {out }} v \simeq \frac{L_{\mathrm{Edd}}}{c}$,

where $\dot{M}_{\text {out }}$ is the mass outflow rate and $L_{\text {Edd }}$ the Eddington luminosity, while the mechanical energy flux is

$\frac{1}{2} \dot{M}_{\text {out }} v^{2} \simeq \frac{L_{\mathrm{Edd}}{ }^{2}}{2 \dot{M}_{\text {out }} c^{2}}$.

These outflows appear to be a widespread phenomenon, not only in currently observed systems such as quasars and ULXs, but also in the growth of supermassive black holes in the centres of galaxies in the past.

\section{OUTFLOWS FROM EDDINGTON- LIMITED ACCRETORS}

I outline here a simple theory of outflows from black holes accreting at rates comparable with the Eddington value

$\dot{M}_{\mathrm{Edd}}=\frac{4 \pi G M}{\eta \kappa c}$.

Here $\eta c^{2}$ is the accretion yield from unit mass, and $\kappa$ is the electron scattering opacity. We assume that the outflow is radial, in a double cone occupying solid angle $4 \pi b$, and has constant speed $v$ for sufficiently large radial distance $r$. I will justify the second assumption later in this Section. Mass conservation implies an outflow density

$\rho=\frac{\dot{M}_{\text {out }}}{4 \pi v b r^{2}}$.

The electron scattering optical depth through the outflow, viewed from infinity down to radius $R$, is

$\tau=\int_{R}^{\infty} \kappa \rho \mathrm{d} r=\frac{\kappa \dot{M}_{\mathrm{out}}}{4 \pi v b R}$.

From (3] 5e get

$\tau=\frac{1}{2 \eta b} \frac{R_{\mathrm{s}}}{R} \frac{c}{v} \frac{\dot{M}_{\mathrm{out}}}{\dot{M}_{\mathrm{Edd}}}$.

Defining the photospheric radius $R_{\mathrm{ph}}$ as the point $\tau=1$ gives

$\frac{R_{\mathrm{ph}}}{R_{\mathrm{s}}}=\frac{1}{2 \eta b} \frac{c}{v} \frac{\dot{M}_{\mathrm{out}}}{\dot{M}_{\mathrm{Edd}}} \simeq \frac{5}{b} \frac{c}{v} \frac{\dot{M}_{\mathrm{out}}}{\dot{M}_{\mathrm{Edd}}}$ 
where we have taken $\eta \simeq 0.1$ at the last step. If $b \leq 1, v / c<1$ we see that $R_{\mathrm{ph}}>R_{\mathrm{s}}$ for any outflow rate $\dot{M}_{\text {out }}$ of order $\dot{M}_{\text {Edd }}$, that is, such outflows are Compton thick. If instead $b<<1$, photons typically escape from the side of the outflow rather than making their way radially outwards through all of it. Almost all of the photons escape in this way within radial distance $r=R_{\perp}$ where the optical depth across the flow

$\tau_{\perp} \simeq \kappa \rho(r) b^{1 / 2} r$

is of order unity. Thus

$\frac{R_{\perp}}{R_{\mathrm{s}}}=\frac{1}{2 \eta b^{1 / 2}} \frac{c}{v} \frac{\dot{M}_{\mathrm{out}}}{\dot{M}_{\mathrm{Edd}}} \simeq \frac{5}{b^{1 / 2}} \frac{c}{v} \frac{\dot{M}_{\mathrm{out}}}{\dot{M}_{\mathrm{Edd}}}$,

and we again conclude that the outflow is Compton thick for $\dot{M}_{\text {out }} \sim \dot{M}_{\text {Edd }}$.

This evidently implies that much of the emission from such objects will be thermalized and observed as a softer spectral component (see eq 20]below). The observed harder X-rays must presumably either be produced near the skin of the outflow (i.e. at moderate $\tau$ ), or result from shocks within the outflow. In both cases their total luminosity must be lower than that of the thermalized soft component.

We now investigate how the outflow is driven. Since the wind is Compton thick most of the photons have scattered and thus on average given up their original momentum to the outflow. Outside the radius $R_{\mathrm{ph}}$ or $R_{\perp}$ the photons decouple from the matter and there is no more acceleration. This justifies our assumption that $v$ is constant for large $r$, and it is self-consistent to use the assumption to integrate inwards to $R_{\mathrm{ph}}$ or $R_{\perp}$.

To ensure that the matter reaches the escape speed we assume that the radii $R_{\mathrm{ph}}, R_{\perp}$ to lie close to the escape radius $R_{\text {esc }}$, i.e.

$R_{\mathrm{ph}}, R_{\perp} \simeq R_{\mathrm{esc}}=\frac{c^{2}}{v^{2}} R_{\mathrm{s}}$.

If instead the photosphere is outside the escape radius, i.e. there is substantial photon trapping, one would insert an optical depth factor $\tau$ on the rhs. The quasar observations referred to above, and the normalization of the $M_{\mathrm{BH}}-\sigma$ relation derived below both suggest that $\tau \simeq 1$, and $\mathrm{I}$ adopt this value in the rest of this article.

From (10) and (7) 9) we find

$\frac{v}{c} \simeq \frac{2 \eta f \dot{M}_{\mathrm{Edd}}}{\dot{M}_{\mathrm{out}}}, \quad R_{\mathrm{ph}, \perp} \simeq\left(\frac{\dot{M}_{\mathrm{out}}}{2 \eta f \dot{M}_{\mathrm{Edd}}}\right)^{2}$

where $f=b, b^{1 / 2}$ in the two cases $b<1, b<<1$. We can write these formulae more compactly as

$\frac{v}{c}=\frac{2 f L_{\mathrm{Edd}}}{\dot{M}_{\text {out }} c^{2}}$

$\frac{R_{\mathrm{ph}, \perp}}{R_{\mathrm{S}}}=\left[\frac{\dot{M}_{\mathrm{out}} c^{2}}{2 f L_{\mathrm{Edd}}}\right]^{2}$,

We note that $f \sim 1$ except for very narrowly collimated outflows $\left(b<10^{-2}\right)$.

An immediate consequence of (12) is

$\dot{M}_{\text {out }} v=2 f \frac{L_{\mathrm{Edd}}}{c}$,

i.e. the momentum flux in the wind is always of the same order as that in the Eddington-limited radiation field, as expected for an Compton thick wind driven by radiation pressure. The energy flux (mechanical luminosity) of the wind is lower than that of the radiation field by a factor of order $v / c$ :

$\dot{M}_{\text {out }} \frac{v^{2}}{2}=\frac{v}{c} f L_{\mathrm{Edd}}=\frac{2\left(f L_{\mathrm{Edd}}\right)^{2}}{\dot{M}_{\text {out }} c^{2}}$.

\section{THE BLACKBODY COMPONENT}

Since the outflow is Compton thick for $\dot{M}_{\text {out }} \sim$ $\dot{M}_{\text {Edd }}$, much of the accretion luminosity generated deep in the potential well near $R_{\mathrm{s}}$ must emerge as blackbody-like emission from it. If $b \sim 1$ the quasi-spherical radiating area is

$A_{\text {phot }}=4 \pi b R_{\mathrm{ph}}^{2}$

If instead $b<<1$ the accretion luminosity emerges from the curved sides of the outflow cones, with radiating area

$A_{\perp}=2 \pi R_{\mathrm{ph}}^{2} b^{1 / 2}$

We can combine these two cases as

$A_{\mathrm{ph}, \perp}=4 \pi g\left[\frac{\dot{M}_{\mathrm{out}} c^{2}}{2 L_{\mathrm{Edd}}}\right]^{4} R_{\mathrm{s}}{ }^{2}$ 
with $g(b)=1 / b, 1 / 2 b^{1 / 2}$ in the two cases. Again $g \sim 1$ unless $b<10^{-2}$, so the areas are similar in the two cases. However we note that the radiation patterns differ. In particular if $b$ is small radiation is emitted over a wider solid angle than the matter. Numerically we have

$A_{\mathrm{ph}, \perp}=2 \times 10^{29} g \dot{M}_{1}^{4} M_{8}^{-2} \mathrm{~cm}^{2}$,

where $\dot{M}_{1}=\dot{M}_{\text {out }} /\left(1 \mathrm{M}_{\odot} \mathrm{yr}^{-1}\right), M_{8}=M / 10^{8} \mathrm{M}_{\odot}$. The effective blackbody temperature is

$T_{\text {eff }}=1 \times 10^{5} g^{-1 / 4} \dot{M}_{1}^{-1} M_{8}^{3 / 4} \mathrm{~K}$.

Clearly such a component is a promising candidate for the soft excess observed in many AGN and ULXs.

\section{THE $M_{\mathrm{BH}}-\sigma$ RELATION}

It is now widely accepted that the centre of every galaxy contains a supermassive black hole. The close observational correlation (Ferrarese \& Merritt, 2000; Gebhardt et al., 2000; Tremaine et al., 2002) between the mass $M$ of this hole and the velocity dispersion $\sigma$ of the host bulge strongly suggests a connection between the formation of the black hole and of the galaxy itself.

The outflows considered above must have an important effect on this problem. We know that most of the mass of the nuclear black holes is assembled by luminous accretion (Soltan 1982; Yu \& Tremaine, 2002). It seems likely that the rate at which mass tries to flow in towards the central black hole in a galaxy is set by conditions far from the hole, for example by interactions or mergers with other galaxies. It is quite possible therefore that super-Eddington conditions prevail for most of the time that the central black hole mass is being built up. Evidently the resulting Eddington thrust (14) can have an important effect on the host galaxy. Unlike luminous energy, a large fraction of a mechanical energy flux like (15) is likely to be absorbed within the galaxy. To reach its present mass the black hole in PG1211+143 could have accreted at a rate comparable to its current one for $\sim 5 \times 10^{7} \mathrm{yr}$. During that time, an outflow like the observed one could have deposited almost $10^{60} \mathrm{erg}$ in the host galaxy. This exceeds the binding energy $\sim 10^{59}$ erg of a bulge with $10^{11} \mathrm{M}_{\odot}$ and $\sigma \sim 300 \mathrm{~km} \mathrm{~s}^{-1}$.

Ideas presented by Silk and Rees (1998, henceforth SR98) and also considered by Haehnelt et al. (1998), Blandford (1999) and Fabian (1999) are relevant here. These authors envisage a situation in which the initial black holes formed with masses $\sim 10^{6} \mathrm{M}_{\odot}$ before most of the stars. Accretion on to these black holes is assumed to produce outflow, which interacts with the surrounding gas. Without a detailed treatment of the outflow from a supercritically accreting black hole, SR98 used dimensional arguments to suggest a relation between $M$ and $\sigma$. However this still has a free parameter. Given the simple relation (14) one can now remove this freedom. The situation turns out to resemble a momentum-driven stellar wind bubble. Modelling this gives an $M_{\mathrm{BH}}-\sigma$ relation devoid of free parameters, and remarkably close to the observed relation.

\subsection{Black hole wind bubbles}

SR98 modelled a protogalaxy as an isothermal sphere of dark matter. If the gas fraction is $f_{g}=$ $\Omega_{\text {baryon }} / \Omega_{\text {matter }} \simeq 0.16$ (Spergel et al., 2003) its density is

$\rho=\frac{f_{g} \sigma^{2}}{2 \pi G r^{2}}$

where $\sigma$ is assumed constant. The gas mass inside radius $R$ is

$M(R)=4 \pi \int_{0}^{R} \rho r^{2} \mathrm{~d} r=\frac{2 f_{g} \sigma^{2} R}{G}$

I now assume that mass flows towards the central black hole at some supercritical rate $\dot{M}_{\text {acc }}$, and thus exerts a momentum flux (14) on the surrounding gas, sweeping it up into a shell. As is well known from the theory of stellar wind bubbles (e.g. Lamers \& Casinelli 1999) this shell is bounded by an inner shock where the wind velocity is thermalized, and an outer shock where the surrounding gas is heated and compressed by the wind. These two regions are separated by a contact discontinuity. The shell velocity depends on whether the shocked wind gas is able to cool ('momentum-driven' flow) or not ('energydriven' flow). In the absence of a detailed treat- 
ment of a quasar wind, SR98 appear to have assumed the second case. In fact for the supercritical outflows envisaged here it is easy to show that the bubble is efficiently Compton cooled, and thus momentum-driven instead (King, 2003)

\subsection{The $M_{\mathrm{BH}}-\sigma$ relation}

The speed $v_{m}$ of the momentum-driven shell now follows from the standard wind bubble argument. At sufficiently large radii $R$ the sweptup shell mass $M(R)$ is much larger than the wind mass, and the shell expands under the impinging wind ram pressure $\rho v^{2}$ (this characterizes momentum-driven flows; in an energy-driven flow the thermal pressure of the shocked wind gas is dominant, while in a supernova blast wave the momentum injection is instantaneous rather than continuous). The shell's equation of motion is thus

$\frac{\mathrm{d}}{\mathrm{d} t}[M(R) \dot{R}]=4 \pi R^{2} \rho v^{2}=\dot{M}_{\text {out }} v=\frac{L_{\mathrm{Edd}}}{c}$

where we have used first the mass conservation equation for the quasar wind, and then (14) to simplify the rhs. Integrating this equation for $\dot{R}$ with the final form of the rhs gives

$M(R) \dot{R}=\frac{L_{\mathrm{Edd}}}{c} t$

where I have neglected the integration constant as $M(R)$ is dominated by swept-up mass at large $t$. Using (22) for $M(R)$ and integrating once more gives

$R^{2}=\frac{G L_{\mathrm{Edd}}}{2 f_{g} \sigma^{2} c} t^{2}$,

where again we may neglect the integration constant for large $t$. We see that in the snowplow phase the shell moves with constant velocity $v_{m}=R / t$, with

$v_{m}^{2}=\frac{G L_{\mathrm{Edd}}}{2 f_{g} \sigma^{2} c}$.

Note that this velocity is larger for higher $L_{\mathrm{Edd}}$, i.e. higher black hole mass. This solution holds if the shell is inside the cooling radius $R_{c}$; outside this radius the shell speed eventually increases to the energy-driven value $v_{e}$, which also grows with $M_{\mathrm{BH}}$.
I now consider the growth of the black hole mass by accretion. Initially the mass is small, inflow is definitely supercritical, and even the energy-driven shell velocity would be smaller than the escape velocity $\sigma$. No mass is driven away, and accretion at a rate $\dot{M}_{\text {Edd }}$ can occur efficiently. However as the black hole grows, we eventually reach a situation in which $v_{e}>\sigma>v_{m}$. Further growth is now only possible until the shell reaches $R_{c}$, and then only until the point where $v_{m}=\sigma$. Thus given an adequate mass supply, e.g. through mergers, the final black hole mass is given by setting $v_{m}=\sigma$ in (26). Thus we find the relation

$M_{\mathrm{BH}}=\frac{f_{g}}{2 \pi} \frac{\kappa}{G^{2}} \sigma^{4} \simeq 1.5 \times 10^{8} \sigma_{200}^{4} \mathrm{M}_{\odot}$.

This is remarkably close to the observed relation (Tremaine et al., 2000). The relation also has no free parameter, unlike earlier derivations (SR98; Haehnelt et al., 1998; Blandford 1999; Fabian 1999) If $v$ had been larger by an optical depth factor $\tau>1$ (i.e. $R_{\mathrm{ph}}, R_{\perp}$ were outside the escape radius $R_{\text {esc }}$ ) a factor $1 / \tau$ would have appeared on the rhs. Taking this factor $\sim 1$ as implicitly done in deriving eqn (10) above agrees with the observed outflow velocities $\sim\left(G M / R_{\mathrm{ph}}\right)^{1 / 2}$ in supercritically accreting quasars.

This derivation of the $M_{\mathrm{BH}}-\sigma$ relation implies that the central black holes in galaxies gain most of their mass in phases of super-Eddington inflow. As relatively few AGN are observed in such phases, these must either be obscured (cf Fabian, 1999) or at high redshift. It appears then that those quasars which are apparently now accreting at such rates (Pounds et al., 2003a,b; Reeves et al., 2003) are laggards in gaining mass. This idea agrees with the general picture that these objects - all narrow-line quasars - are super-Eddington because they have low black-hole masses, rather than unusually high mass inflow rates.

\section{ULX NEBULAE}

We have seen that outflows from Eddingtonlimited AGN can have a profound effect on their surroundings, in the form of the $M_{\mathrm{BH}}-\sigma$ relation. In the stellar-mass context, ULXs are the most supercritically accreting objects, and one would 
naturally expect a similar effect here too. Indeed, many ULXs are observed to lie in the centres of unusually large and bright emission nebulae. These have in the past been variously interpreted as supernova/hypernova remnants (cf Roberts et al, 2003) or photoionized nebulae (Pakull \& Mirioni, 2003). However it is clear from the work of the last Section that a more likely explanation of the unusual energetics of these objects is as bubbles blown in the ISM by an Eddington outflow from the ULX. Pakull \& Mirioni (2003) indeed note that the nebulae are probably shock excited. This interpretation means that the nebulae are powered by the accumulated kinetic energy of the outflow that was dissipated over the gas cooling time in the nebula (or the ULX lifetime if this is shorter). In particular there is no requirement that the radiation currently emitted from the ULX should power the emission nebula. The nebulae do not provide arguments against anisotropic emission from this source: even if the wind is anisotropic, it is probable that it would have precessed many times over the ULX's lifetime, and thus left a roughly spherical nebula, as observed. One cannot therefore use ULX nebulae to argue against the interpretation of ULXs as anisotropically emitting stellar-mass X-ray binaries (cf King et al., 2001).

\section{CONCLUSION}

Mass outflows from Eddington-limited accreting compact objects appear to be a very widespread phenomenon. They have major implications for quasars and galaxy formation, and for ULXs. Some of these are barely explored as yet, and the field promises to be fruitful.

I thank Andy Fabian, Jim Pringle and James Binney for illuminating discussions. Some of the work reported here was carried out at the Center for Astrophysics, and I thank members of its staff, particularly Pepi Fabbiano and Martin Elvis, for stimulating discussions and warm hospitality. I gratefully acknowledge a Royal Society Wolfson Research Merit Award.

\section{REFERENCES}

1. Blandford, R.D., 1999, in Galaxy Dynamics, ASP Conference Series 182, eds. D.R Merritt, M. Valluri, J.A. Sellwood (San Francisco: ASP), 87

2. Fabian, A.C., 1999, MNRAS 308, 39

3. Ferrarese, L., Merritt, D., 2000, ApJ 539, L9

4. Gebhardt, K., Bender, R., Bower, G., Dressler, A., Faber, S.M., Filippenko, A..V., Green, R., Grillmair, C., Ho, L.C., Kormendy, J., Lauer, T.R., Magorrian, J., Pinkney, J., Richstone, D. and Tremaine, S., 2000, ApJ 539, L13

5. Haehnelt, M., Natarajan, P., Rees, M.J., 1998, MNRAS 300, 817

6. King, A.R., Davies, M.B., Ward, M.J., Fabbiano, G., Elvis, M., 2001, ApJ, L552, 109

7. King, A.R., 2003 ApJ Lett, in press (astroph-ph/0308342)

8. King, A.R., Pounds, K.A., 2003, MNRAS, in press (astro-ph/0305541)

9. Lamers, H.J.G.L.M., Casinelli, J.P., 1999, Introduction to Stellar Winds, Cambridge University Press, Cambridge, Ch. 12

10. Pakull, M.W., Mirioni, L., 2003, ReVMexAA 15, 197

11. Pounds, K.A., Reeves, J.N., King, A.R., Page, K.L., O'Brien, P.T., Turner, M.J.L., 2003a, MNRAS in press (astro-ph/0303603)

12. Pounds, K.A., King, A.R., Page, K.L., O'Brien, P.T., 2003b, MNRAS, in press, astr-ph/0305571

13. Reeves, J.N., O'Brien, P.T., Ward, M.J., 2003, ApJ, 593, 65

14. Roberts, T.P., Goad, M.R., Ward, M.J., Warwick, R.S., 2003, MNRAS 342, 709

15. Soltan, A., 1982, MNRAS, 200, 115

16. Spergel et al., 2003, ApJ, in press (astro-ph/0302209))

17. Silk, J., Rees, M.J., 1998, A\&A, 331, L1 (SR98)

18. Tremaine, S., Gebhardt, K., Bender, R., Bower, G., Dressler, A., Faber, S.M., Filippenko, A.V., Green, R., Grillmair, C., Ho, L.C., Kormendy, J., Lauer, T., Magorrian, J., Pinkney, J., Richstone, D., 2002, ApJ 574, 740 
19. Yu, Q., Tremaine, S., 2002, MNRAS 335, 965 